\title{
Imparare l'imperativo a partire dalla mindfulness. Un project work realizzato in una scuola di Barcellona
}

\author{
Daniela Palmeri \\ Euroaula Escuela Universitaria de Turismo di Barcellona \\ daniela.palmeri@euroaula.com
}

\begin{abstract}
Questo saggio analizza un project work di italiano come LS (livello linguistico A1/A2), svolto in una scuola secondaria di primo grado di Barcellona, Institut Escola Artístic Oriol Martorell. Il project work si è basato sulla mindfulness a partire dall'approccio comunicativo e anche da quello umanistico-affettivo. La prima parte dello studio indaga alcuni concetti chiave (competenza comunicativa; task e project work; mindfulness). La seconda parte presenta il caso di studio in cui vengono applicate la mindfulness e la meditazione per imparare a rilassarsi attraverso l'uso dell'imperativo. Il project work ha permesso di perseguire contemporaneamente l'acquisizione di abilità linguistiche e anche personali e sociali. I risultati mostrano come la mindfulness applicata all'apprendimento dell'italiano come LS consenta di potenziare l'autoconsapevolezza e anche la creatività, attraverso l'apprendimento significativo.
\end{abstract}

Parole chiave: Italiano LS; approccio comunicativo e umanistico-affettivo; project work; mindfulness; imperativo.

\section{Abstract. Learning the imperative through mindfulness. A project work carried out in a school in Barcelona.}

This essay aims to analyze a project-work of Italian as a Foreign Language (IFL), carried out with a group of A1/A2-skilled students at a secondary school in Barcelona, Institut Escola Artístic Oriol Martorell. The project work was based on mindfulness from a communicative and humanistic approach. The first part of the study investigates some key concepts (communicative competence; task and project-work; mindfulness). The second part introduces a case study where mindfulness and meditation are applied, jointly with the use of the imperative mood for advising to relax. The project-work enables to pursue simultaneously language acquisition and personal and social skills. The results show that mindfulness applied to IFL learning allows to sharpen self-awareness and creativity through a meaningful learning.

Keywords: Italian FL; humanistic language teaching; project work; mindfulness; imperative mood. 


\section{Introduzione}

Nel presente saggio analizzo un'esperienza d'insegnamento dell'italiano come LS, svolta durante il primo trimestre del 2016, presso l'Institut Escola Artístic Oriol Martorell di Barcellona, ${ }^{1}$ istituto pubblico comprensivo di scuola primaria (Escuela Primaria) e anche di scuola secondaria di primo e secondo grado (Escuela Secundaria Obligatoria, conosciuta come ESO, e Bachillerato). ${ }^{2}$ L'Istituto Martorell realizza un curricolo formativo integrato fra indirizzo ordinario e indirizzo potenziato dalla pratica dello strumento musicale. Si tratta di una delle pochissime scuole della Catalogna in cui si insegna italiano come lingua straniera (due ore alla settimana nel terzo corso e un'ora nel quarto corso della ESO).

In questa scuola ho svolto un project work intitolato Impara, medita e rilassati su mindfulness e imperativo, nella terza classe della ESO (età media: quattordici anni), il cui livello linguistico è quello di un A1-A2 del Quadro Comune Europeo di Riferimento (d'ora in poi QCER). Il progetto si è ispirato ai principi della mindfulness a partire da un approccio comunicativo e anche umanistico-affettivo. Mediante l'uso di tecniche meditative, la mindfulness consente di potenziare l'attenzione consapevole e la creatività e anche di superare lo stress che può provocare l'apprendimento della LS. L'intervento didattico ha puntato sullo sviluppo e sul potenziamento delle abilità linguistiche di base (espressione scritta e orale e comprensione scritta e orale) attraverso una metodologia eclettica nell'insegnamento della grammatica, volta sempre a promuovere un apprendimento attivo e significativo della LS. Grazie al project work, gli alunni hanno potuto acquisire l'uso delle forme imperativali della seconda persona singolare per dare consigli su come rilassarsi.

Nell'era della globalizzazione e delle tecnologie digitali, la didattica per progetti può contribuire ancora a superare la sfida della complessità mettendo l'accento sulla relazione insegnante/discente. La sperimentazione didattica attraverso il project work ha cercato di rispondere ai bisogni di educazione linguistica degli alunni di questa scuola che manifesta una considerevole apertura ai percorsi culturali e artistici trasversali, dato l'indirizzo musicale del curricolo.

Per quanto riguarda la struttura generale di questo saggio, nelle prima parte si analizzano alcune teorie e concetti chiave: l'approccio comunicativo, i task e la didattica progettuale (sezione 1); le origini del project work e la sua applicazione alla LS (sezione 2); l'approccio umanistico-affettivo (sezione 3); i principi fondamentali della mindfulness e le sue potenzialità nell'ambito dell'educazione linguistica (sezione 4). Nella seconda parte invece si spiega come funziona il curricolo della scuola in relazione alla competenza comunicativa (sezione 5) e si sviscerano i principi e la struttura del project work Impara,

1. In questa scuola, ho realizzato il tirocinio durante il Máster de Formación del Profesorado (svolto presso l'Università di Barcellona, nell'a.a. 2015-2016), equivalente all'abilitazione per l'insegnamento dell'italiano come LS.

2. La ESO dura quattro anni ed è una tappa scolastica obbligatoria, mentre il Bachillerato consiste in un biennio specialistico post-obbligatorio. 
medita e rilassati (sezioni 6-7). L'appendice finale presenta anche una selezione del materiale didattico creato.

\section{Dall'approccio comunicativo al task e al project work}

È ben risaputo che negli anni Sessanta inizia una svolta nell'epistemologia che si ripercuote anche nella didattica delle lingue. ${ }^{3}$ L'approccio comunicativo viene teorizzato da Dell Hymes (1971) a partire dal concetto di "competenza comunicativa", che pone l'accento sulla comunicazione come mezzo e fine dell'apprendimento in una prospettiva sociale legata al contesto di uso della lingua. Insegnare una lingua implica il difficile lavoro di insegnare a comunicare e pertanto ingloba anche fattori extralinguistici e socioculturali. L'approccio comunicativo sostituisce quindi l'insegnamento basato sulla grammatica e può servirsi di metodi diversi, fra i quali il più riconosciuto è quello nozionale-funzionale. Grazie a vari arricchimenti e approfondimenti, ${ }^{4}$ la teoria comunicativa continua a essere viva e costituisce ancora un cardine della glottodidattica, come si può ben notare nelle idee principali del QCER, che costituisce una base di riferimento unica per l'apprendimento e l'insegnamento delle lingue in Europa (Consiglio d'Europa, 2002). ${ }^{5}$ Tuttavia oggigiorno sarebbe più opportuno parlare di approcci comunicativi al plurale.

L'approccio per compiti o task (dall'inglese Task-Based Language Teaching) rappresenta un'evoluzione dell'approccio comunicativo e si diffonde alla fine degli anni Ottanta, mettendo in evidenza l'importanza di procedere attraverso compiti che promuovano un uso reale della lingua (Breen, 1987). Pertanto il task pone l'accento soprattutto sull'apprendimento basato sull'azione. ${ }^{6}$ Lo studioso David Nunan (2004) sottolinea come il task si focalizzi soprattutto sul significato piuttosto che sulla forma.

Sarebbe impossibile nello spazio di queste pagine ripercorrere tutti gli studi in questo ambito; ${ }^{7}$ tuttavia metterò in risalto alcuni punti chiave nella bibliografia spagnola e italiana, cercando di sottolineare come avvenga il passaggio dal compito al project work.

3. Sull'influenza degli anni Sessanta nella glottodidattica, cfr. Balboni (2017).

4. Ricordo solamente che il modello di Hymes viene poi ripreso e approfondito da Canale $\&$ Swain (1980) che distinguono tre aspetti importanti: le competenze grammaticale, sociolinguistica e strategica. In seguito Canale (1983) ritorna su questo modello e aggiunge anche il concetto di competenza discorsiva. Un altro modello importante che prende spunto da quello di Hymes è sicuramente quello di Bachman (1990), il quale parla anche di competenza organizzativa e pragmatica.

5. Inoltre uno dei primi documenti del Consiglio d'Europa che preannuncia la svolta comunicativa è il Livello Soglia, che riassume le abilità e i contenuti del livello B1 e funziona come strumento di progettazione curricolare. Nel caso del Livello Soglia per l'italiano, cfr. Galli De’ Paratesi (1981).

6. Un recente e interessante articolo sul tema dei task e anche sull'approccio orientato all'azione è quello di Birello, Odelli \& Vilagrasa (2017).

7. Un libro che fa luce sui molteplici approcci e i metodi della glottodidattica è Richards $\&$ Rodgers (2001). 
Nell'ambito della bibliografia in spagnolo, un libro fondamentale è Project Work Step by Step, scritto a quattro mani da Ramon Ribé e Nuria Vidal nel 1993. Ribé \& Vidal (1993, pp. 2-3) distinguono fra compiti di prima, seconda e terza tipologia: il primo tipo di task è semplice e quasi meccanico, il secondo prevede uno sviluppo cognitivo e il terzo uno sviluppo creativo. Ribé \& Vidal (1993, p. 4) spiegano che i task di seconda e terza tipologia possono essere definiti come parte del lavoro a progetto perché contribuiscono allo sviluppo personale degli alunni. In questo modo, si stabilisce un interessante collegamento fra il task e il project work a partire dallo sviluppo della dimensione creativa. In un saggio successivo intitolato Tramas creativas, Ribé (1997) afferma che i task di seconda e terza tipologia hanno a che vedere con un tipo di experiential learning o "apprendimento vivenziale" e propongono un lavoro complesso sulla lingua a partire dal learning by doing, "facendo si impara". In questo senso una costellazione di task può essere definita come una "trama creativa" se fomenta lo sviluppo integrale della persona.

Allo stesso tempo, nell'ambito della bibliografia italiana, lo studioso Gabriele Ridarelli (1998, p. 173) sottolinea che "un progetto può essere anche definito come un insieme di compiti o task (per la maggior parte comunicativi) finalizzati ad uno scopo generale, con riscontro nel mondo reale". Con queste parole, si riassume bene il senso di un project work: il procedere per fasi $\mathrm{e}$ attività e l'apertura al mondo reale.

Ne La didattica per progetti, curato da Franca Quartapelle (2005 [1999]), si mette in risalto l'importanza di una didattica progettuale basata sul fare e sui bisogni individuali e sociali degli alunni. In un certo senso, tutte le tesi sul project work sottolineano l'importanza del dialogo fra il docente e la classe. Si potrebbe dire che la caratteristica di base di un progetto è il "fare dialogico", come afferma Ceragioli (2005, p. 107). Attraverso la didattica laboratoriale si permette agli studenti di diventare i protagonisti attivi del loro processo di apprendimento. Detto questo, quindi, il lavoro a progetto può essere visto come una modalità del lavoro per compiti all'interno dell'approccio comunicativo. Se il lavoro a progetto sviluppa la creatività del discente allora si realizza una trama di tipo creativo.

\section{Educazione a progetto e acquisizione della lingua}

Quali sono le radici del project work? L'educazione a progetto nasce all'inizio del XX secolo in seno alla pedagogia pragmatista americana, grazie agli studi di John Dewey, che nel 1916 conia l'espressione learning by doing con cui propone l'abolizione della suddivisione per materie e il lavoro per progetti a partire da tematiche considerate interessanti. Il discepolo e collaboratore di Dewey, William Heard Kilpatrick, nel suo saggio The project Method (1918) approfondisce il tema dell'apprendimento attivo ed elabora il "metodo dei progetti" sottolineando l'importanza che gli alunni apprendano attraverso esperienze concrete. 
In un certo senso, possiamo considerare queste teorie come un modello pedagogico propedeutico, poi ripreso anche dalla didattica delle lingue. La studiosa Diana Fried-Booth (1986), spiega come funziona un progetto nei corsi di inglese come LS centrandosi su tre punti importanti: autenticità, autonomia e motivazione. Qualsiasi lavoro a progetto deve rifarsi alla pedagogia attiva e puntare su questi tre obiettivi fondamentali, che hanno come centro il discente. L'autenticità si riferisce proprio all'uso della lingua attraverso un'esperienza reale.

Secondo gli autori tedeschi Legutke \& Thomas (1991) il project work nella LS parte non solo dagli approcci comunicativi, ma anche da una concezione olistica e gestaltica dell'apprendimento. ${ }^{8}$ I punti più importanti di un buon progetto sono: l'esperienza, il processo, la classe cooperativa, la dinamica individuo/gruppo (Legutke \& Thomas, 1991, p. 203).

The process orientation of the classroom commits its participants to a focus on 'experience' as an essential and substantial element of their learning. (Legutke \& Thomas, 1991, p. 214)

In effetti in tutti gli studi sul project work ritorna l'accento sull'esperienza che rende possibile l'apprendimento di un sapere pratico attraverso la scoperta.

Rispetto alla classificazione delle tipologie di progetto, Henry (1994) distingue progetti strutturati, semistrutturati e non strutturati. Questa distinzione dipende dalla libertà lasciata agli alunni di scegliere il tema e di ricercare il materiale utile (Henry, 1994, pp. 13-16). Nel caso del progetto non strutturato gli alunni hanno massima libertà di scelta e autodeterminazione, mentre negli altri casi l'insegnante ha scelto già il tema su cui lavorare.

Inoltre Fredricka Stoller (1997) spiega anche che la maggior parte dei progetti non solo utilizzano l'esperienza come contenuto, ma anche come risultato finale, che coincide sempre con qualcosa di autentico. Secondo Stoller (1997), i punti importanti di questa metodologia sono: il project work si basa sui contenuti (content learning basato sul mondo reale e sugli interessi degli alunni); si centra sullo studente; promuove un apprendimento cooperativo; finisce sempre con la realizzazione di un prodotto finale (anche se non è questo il suo obiettivo principale). ${ }^{9}$ Gli alunni possono partecipare a una negoziazione aperta sul programma e sul risultato finale. Pertanto, un altro punto nodale di qualsiasi progetto è sicuramente l'apprendimento cooperativo. È evidente che il lavoro a progetto permette di lavorare su varie dimensioni: comunicativolinguistica; affettivo-motivazionale, sociale e di gruppo.

8. Legutke \& Thomas (1991, pp. 160-165) distinguono tre tipi di progetto che è possibile sviluppare nei corsi di lingua: 1) encounter project, incontri con parlanti nativi; 2) text project, in cui gli alunni possono elaborare dei testi di vario tipo; 3) class correspondence project, in cui si promuovono incontri attraverso corrispondenze.

9. Cfr. anche Alan \& Stoller (2005). 


\section{L'importanza dell'approccio umanistico-affettivo nella LS}

L'approccio umanistico-affettivo ha inizio negli anni Sessanta con il testo di psicodidattica Toward a Theory of Instruction di Jerome Bruner (1966) e anche con il saggio di psicologia Freedom to Learn dello psicologo Carl Rogers (1969). In entrambi i saggi, la sfera affettiva assume un valore centrale a partire da una visione umanistica della relazione insegnante-alunno. In questo ambito è senza dubbio fondamentale il concetto di apprendimento "significativo", che è diametralmente opposto all'apprendimento meccanico ed enfatizza l'importanza della costruzione di significati nel processo educativo. ${ }^{10}$

Che peso ha la sfera affettiva nell'acquisizione della lingua straniera? La dimensione affettiva viene messa in luce da Stephen Krashen (1981), che rifiuta il concetto di apprendimento e lo sostituisce con quello di "acquisizione", sottolineando in questo modo l'importanza dei meccanismi subconsci nell'interiorizzazione della lingua straniera. Krashen parla di un filtro affettivo, una sorta di meccanismo di autodifesa, che può provocare un blocco e deve essere superato attraverso un clima di fiducia in classe.

Già a partire dagli anni Settanta la glottodidattica assorbe queste teorie e crea dei metodi specifici: ad esempio il Total Physical Response (TPR), la suggestopedia, il Silent Way, il Community Language Learning.

Fra tutti questi metodi, il project work che ho proposto nella scuola secondaria ha attinto sia al TPR sia alla suggestopedia. Il metodo TPR, tradotto in italiano come "Risposta Fisica Totale", è stato inventato dallo psicologo James Asher (1969) e sviluppa l'importanza della risposta corporea nell'apprendimento della lingua, soprattutto attraverso l'azione. Nella seconda fase del project work proposto si utilizza questo metodo per favorire un apprendimento fisico e anche ludico della LS. Inoltre, la terza fase del project work, basata sulla meditazione, presenta vari punti in comune con il metodo suggestopedico, fondato dallo psichiatra bulgaro Georgi Lozanov (1978). La suggestopedia prevede l'utilizzo di tecniche di rilassamento, della respirazione ritmica e anche della musica per favorire la concentrazione e la memorizzazione.

Un altro tema di studio fondamentale dell'approccio umanistico-affettivo è il rapporto tra educazione linguistica ed emozioni, ${ }^{11}$ basilare per migliorare la motivazione. Fra gli esponenti più rinomati della glottodidattica umanistica italiana, lo studioso Mario Cardona (2010) mette in evidenza l'importanza di una visione integrata della dimensione emotiva e di quella cognitiva, visto che le memoria emotiva agisce in maniera determinante sull'acquisizione della lingua straniera. ${ }^{12}$ Pertanto, alle emozioni viene riconosciuto un ruolo chiave.

10. Il tema dell'apprendimento significativo è centrale nella psicologia costruttivista. Cfr. Ausubel (1963).

11. Per un approfondimento sul valore delle emozioni, cfr. Goleman (1995).

12. In Italia, la glottodidattica umanistico-affettiva è stata ed è particolarmente produttiva e trova le sue origini già negli studi dello psicologo Renzo Titone (1971) e anche con Giovanni Freddi (1970), il fondatore della scuola veneziana. All'interno del variegato panorama attuale, cfr. Balboni (2002) e Caon (2005). 
Come vedremo, le teorie sulla mindfullness ben si legano a questi studi perché pongono l'attenzione sulla dimensione affettiva ed emozionale.

\section{La mindfulness e la sua applicazione all'educazione linguistica}

Il termine mindfulness deriva da mindful awareness, "coscienza" o "consapevolezza", che a sua volta è la traduzione inglese della parola sati che significa "attenzione consapevole" in pali, la lingua della tradizione buddista. Le origini di questa pratica sono antichissime e si perdono nella millenaria tradizione orientale. In Occidente, a partire dagli anni Settanta lo psicologo statunitense Jon Kabat-Zinn applica i principi di questa corrente all'ambito clinico e fonda presso l'università del Massachussetts la Stress Reduction Clinic, un programma che permette ai pazienti adulti di superare situazioni di dolore, ansia e stress attraverso le tecniche meditative. Nel suo saggio più rinomato, KabatZinn (1990) spiega la validità dell'applicazione terapeutica della mindfulness sia per fronteggiare situazioni dolorose che per migliorare il benessere psicofisico. ${ }^{13}$ Lo strumento principale è la meditazione che può basarsi su semplici esercizi di respirazione, visualizzazioni e anche sulla consapevolezza corporea (body-scan) o sul movimento lento (meditare camminando). ${ }^{14}$

Molti studi recenti mettono in evidenza l'importanza di questi concetti in ambito educativo (Hassed \& Chambers, 2015). I benefici della mindfulness applicati al mondo della scuola sono molti e vanno dalla riduzione dello stress e dell'ansia al miglioramento della capacità di concentrazione e all'incremento della motivazione. ${ }^{15}$

Detto questo, vorrei tuttavia sottolineare che non sono state effettuate molte ricerche sull'applicazione della mindfulness all'insegnamento della LS, nonostante le potenzialità. In un saggio teorico sul tema della mindfulness e della LS, Toni Houston e Paaige Turner (2007) sottolineano come un'acquisizione consapevole della lingua possa smontare molti falsi miti dell'educazione tradizionale. In generale, in questo ambito molti studi partono proprio da una visione sociocognitiva e si concentrano soprattutto su come la mindfulness riesca ad abbassare l'ansia nel processo di apprendimento della LS (Ghanizadeh, Makiabadi \& Abdi Navokhi, 2019; Fallah, 2016; Önem, 2015).

Un saggio molto interessante sul tema è quello di due docenti cinesi, Yang Wang e Chao Liu (2016), i quali introducono la meditazione in un gruppo di studenti di inglese come LS, nella Northeastern University in Cina. Wang e Liu raccontano i risultati positivi di questa esperienza:

13. Per un approfondimento su mindfulness e psicologia, cfr. Baer \& Maffei (2012).

14. Per quanto riguarda l'etimologia, il termine "meditazione" viene dal latino meditatio, "riflessione", e indica un esercizio intellettuale simile alla contemplazione. Le pratiche meditative più antiche risalgono all'induismo vedico e alla mistica indiana. Oggigiorno esistono vari tipi di meditazione: meditazione zen, buddhista, vipassana, trascendentale, dinamica.

15. In effetti, negli Stati Uniti è nata anche la Contemplative Pedagogy, movimento che si basa sul coltivare presenza e attenzione per favorire uno sviluppo olistico (Roeser \& Peck, 2009; Brady, 2007). 
Through mindfulness practices, the EFL students built a learning community and became aware of this comfortable social environment; mindful writing helped students generate new thoughts and become aware of their thinking; mindfulness facilitated the learning process, cultivated creativity and intelligence; mindful cooperative learning provided the students with an opportunity to discover their awareness, learn from others, reflect and think critically. (Wang \& Liu, 2016, pp. 151-152)

Con queste parole, si mette in evidenza la capacità della mindfulness di creare un contesto educativo in cui sia possibile coltivare la consapevolezza critica e anche la creatività in una prospettiva di educazione linguistica.

\section{Il contesto spagnolo: il curricolo e le competenze}

Il curricolo su cui si basa la scuola secondaria in Spagna prevede un lavoro sulle competenze di base per favorire la realizzazione personale e sociale degli alunni (Departament d'Educació, 2007b). In perfetta concordanza con i principi del QCER, le competenze generali del curricolo si propongono di innescare una sorta di catena di apprendimento che si fondi non solo sul sapere, ma anche sul saper fare, saper essere e saper imparare. ${ }^{16}$ Nello specifico, per quanto riguarda l'ambito della LS, nel curricolo delle scuole dell'obbligo si distinguono due aspetti importanti della competenza comunicativa: da un lato la competenza plurilingue e interculturale; dall'altro la competenza comunicativa orale, scritta, audiovisuale e letteraria (Departament d'Educació, 2007b, pp. 3-5).

Bisogna considerare che il curricolo delle scuole dell'obbligo segue uno sviluppo "verticale" basato sulle materie e sui programmi che si snodano lungo gli anni. Invece, la struttura del project work spinge a ripensare e riformulare questa programmazione "verticale" delle discipline. In effetti una scuola che utilizzi la didattica per progetti lavora sulle competenze anche in maniera orizzontale, cioè a partire da tematiche monografiche e interdisciplinari che strutturano una rete di conoscenze. In questo modo si potrebbe superare la frammentazione disciplinare che non solo non è formativa, ma è anche monotona e poco efficace nel caso dell'educazione linguistica a scuola. Le studiose Preti, Bertocchi e Quartapelle (2002) ribadiscono che la crescita a scuola non include solo la dimensione cognitiva, ma anche quella pragmatica ed esistenziale-valoriale. In quest'ottica la didattica progettuale mette in dubbio il curricolo "lineare" a favore invece di curricoli basati sulle competenze e non solo sui saperi (Quartapelle, 2009, p. 13).

Nel caso specifico dell'insegnamento della LS, il project work permette anche di mettere in pratica il concetto di rule of forgetting di Krashen e Terrell (1983) perché si utilizza la lingua straniera dimenticando che l'obiettivo sia imparare la lingua, visto che ci si concentra molto sulla tematica scelta. In un certo senso, si sposta l'interesse dell'apprendente sul contenuto, sul processo

16. Il QCER parla proprio di “competenza esistenziale" nella sezione 5.1.3 (Consiglio d'Europa, 2002). 
e sul prodotto del project work per diminuire l'ansia e favorire un apprendimento creativo.

Il Progetto Educativo dell'Istituto Oriol Martorell ${ }^{17}$ pone in luce l'importanza di potenziare la sensibilità e le capacità artistiche degli alunni, visto che si tratta di un curricolo ad indirizzo musicale (Institut Escola Artístic Oriol Martorell, 2010). Proprio questo fattore mi ha fatto riflettere sulla possibilità di lavorare attraverso un project work in maniera trasversale unendo competenze di vario tipo e dirigendo lo sguardo verso il potenziamento della creatività.

Di seguito, presento due tabelle che sintetizzano le competenze su cui ho lavorato durante il project work. La tabella 1 riformula le competenze di base della ESO, così come compaiono nel documento chiave emesso del Departament d'Educació (2007b). La tabella 2 invece presenta le competenze linguistico-comunicative a partire dal programma del Departament d'Educació (2007a, pp. 3-5) e anche a partire dalla bibliografia di base di riferimento sui programmi di italiano A1-A2 (Spinelli \& Parizzi, 2010; Consiglio d'Europa, 2002).

Tabella 1. Competenze di base della ESO, inserite nel project work Impara, medita e rilassati

Competenza comunicativa linguistica e audiovisuale: sviluppare la conoscenza della lingua; saper utilizzare le tecnologie digitali.

Competenza comunicativa artistico-culturale: saper riconoscere e comprendere testi artistici e culturali; sviluppare la fantasia, l'immaginazione e la creatività.

Competenza metodologica (imparare ad imparare): sviluppare la capacità di imparare.

Competenza personale (autonomia e iniziativa personale): sviluppare l'autonomia e la responsabilità personale.

Competenza convivere e abitare il mondo (conoscenza del mondo): saper interagire con il mondo fisico.

Competenza convivere e abitare il mondo (sociale e civica): saper interagire con gli altri nel gruppo classe.

Tabella 2. Competenze linguistico-comunicative, inserite nel project work Impara, medita e rilassati

Competenza grammaticale: presente indicativo; imperativo informale (forme regolari e irregolari); imperativo informale dei verbi riflessivi con pronome; imperativo negativo; morfologia del nome (plurali irregolari); lessico del corpo, dell'albero, del musicista.

Competenza discorsiva: dare istruzioni e consigli semplici con l'imperativo; produrre brevi testi, scritti con coesione e coerenza.

17. In Spagna, il Progetto Educativo del centro (Proyecto educativo del centro, conosciuto anche con la sigla $P E C$ ) è il documento che definisce l'identità di una scuola e i suoi obiettivi. 
Competenza strategica: prendere parte a una conversazione semplice sulla tematica del rilassamento; saper risolvere problemi comunicativi semplici come i turni di parola.

Competenza sociolinguistica: saper utilizzare un registro linguistico adeguato; imparare a riconoscere situazioni formali e informali.

Competenza plurilingue e interculturale: mettere in relazione lingue e culture diverse a partire dalla propria identità culturale.

Competenza comunicativo-letteraria: comprendere testi letterari semplici a partire dai quali poter sviluppare una visione critica del mondo e di sé stessi.

\section{Punti essenziali del project work Impara, medita, rilassati}

Nel libro La didattica progettuale Catia Mugnani (2011, p. 4) sottolinea l'importanza di applicare non solo in maniera letterale il project work, ma anche in maniera soft, considerando i progetti come "spunti per attività sempre modulabili". Con tale spirito, è nato questo progetto che si è articolato in cinque sessioni: 1) Se una notte d'inverno uno spettatore; 2) Corpo e movimento; 3) La meditazione dell'albero; 4) Consigli per un giovane musicista; 5) Autovalutazione.

La scelta del tema del project work, incentrata sul rilassamento, è sorta a partire dal dialogo instaurato con gli alunni. Prima di iniziare il progetto, avevo chiesto agli studenti come trascorressero il tempo libero, come si rilassassero e se avessero mai meditato: circa un sessanta percento degli alunni aveva già praticato la meditazione. Inoltre, chi non aveva mai meditato si è mostrato curioso e aperto. Molti alunni della scuola soffrono di stress a causa della competitività cui sono sottoposti durante i concerti; per questo motivo, la proposta è stata ben accolta anche dal centro scolastico.

Dal punto di vista grammaticale, il progetto si è imperniato sull'uso delle forme imperativali di seconda persona singolare (costruzione affermativa e negativa "non + infinito") con lo scopo di imparare a dare consigli. Durante il primo semestre gli alunni avevano solamente studiato l'imperativo dei verbi regolari (imperativo che coincide con il presente indicativo). Nel project work si è affrontato anche il tema dei verbi irregolari (dare, fare, stare, andare e dire) e poi anche l'uso dei pronomi e il raddoppiamento fonosintattico. Inoltre, rispetto all'imperativo negativo si sono studiate le forme con e senza pronomi.

L'imperativo costituisce un tema centrale e anche relativamente semplice per la sua somiglianza e coincidenza con altre forme verbali. Tuttavia è anche un tema che richiede un'acquisizione graduale perché presenta un'alta variabilità morfologica. Pertanto è importante organizzare la sequenza di acquisizione delle sue forme in maniera chiara e ordinata, attraverso attività che favoriscano la memorizzazione. Riflettendo sull'imperativo nell'apprendimento della L2, Monica Berretta (1995) mette in evidenza una contraddizione intrinseca.

Lo spunto inziale per la riflessione viene dal palese contrasto fra, da un lato, la basicità nozionale dell'imperativo che farebbe supporre un apprendimento 
abbastanza precoce, e, dall'altro, la costatazione che al contrario l'imperativo è appreso tardi, e con molte difficoltà, dagli stranieri. (Berretta, 1995, p. 333)

In effetti l'acquisizione dell'imperativo è un processo lento e mai immediato, nonostante la sua centralità a livello nozionale.

L'intervento didattico proposto si è sviluppato sotto due grandi ali: da una parte, l'uso dell'imperativo attraverso situazioni reali e significative e, dall'altra, l'educazione alle emozioni. Pertanto, i nodi fondamentali di questo project work sono stati: educazione linguistica ed emozionale; combinazione di obiettivi linguistico-comunicativi e di quelli relativi allo sviluppo personale e sociale; apprendimento significativo e cooperativo. Alla luce delle teorie esaminate, potrei dire che si è trattato di un project work "semistrutturato" (Henry 1994) nel senso che sono stati negoziati solo alcuni momenti del processo.

La valutazione del project work è stata continua e pedagogica e, per concludere, si è realizzata anche un'autovalutazione attraverso una scheda specifica. Alla fine di ogni sessione era presente anche una fase metacognitiva con lo scopo di riflettere sull'apprendimento della lingua in maniera attiva.

In un saggio molto suggestivo sulla didattica per progetti, Quartapelle afferma:

Non si impara a "elaborare e realizzare progetti" se non applicandosi a farlo, con una metodologia di progetto che superi la logica propria della didattica trasmissiva e consenta di realizzare un apprendimento attivo, legato alla soluzione di problemi che abbiano senso sia dal punto vista del contesto sociale in cui si collocano sia da quello delle esigenze dell'apprendente. (Quartapelle, 2009, p. 9)

In effetti, un progetto deve presentare un certo legame sia con il contesto sociale in cui si produce, sia con le necessità psico-affettive degli alunni. Solo a partire da questo, si può uscire fuori dalla logica ripetitiva e meccanica dell'apprendimento passivo.

\section{Procedura del project work e piano delle attività}

Di seguito si illustrano le fasi più importanti del project work mettendo in evidenza obiettivi, struttura e metodo utilizzati.

I FASE: Se una notte d'inverno uno spettatore (2 ore)

Presentazione generale. Dopo aver presentato il macrotema e le fasi del progetto, sono stati esposti gli obiettivi generali: creare un prodotto individuale (un testo di scrittura libera dopo la meditazione della III fase) e di gruppo (un decalogo di consigli per un musicista nella IV fase). Gli alunni hanno svolto un'attività di warm-up attraverso un dibattito sul rilassamento e il tempo libero. Ognuno ne ha parlato prima con il compagno e poi in gruppo.

La letteratura a scuola. Questa parte del project work si è basata sull'uso della letteratura, risorsa straordinaria per l'educazione linguistica ed emozionale, ${ }^{18}$

18. Sull'uso della letteratura nell'insegnamento della LS e della L2, cfr. Ardissino \& Stroppa (2001). 
alla quale spesso non viene data la dovuta importanza nei corsi di LS, soprattutto nei livelli iniziali. La sperimentazione con la letteratura è partita dall'analisi dell'incipit del romanzo di Italo Calvino Se una notte d'inverno un viaggiatore $e^{19}$ che contiene molti consigli all'imperativo destinati a un ipotetico lettore. ${ }^{20}$ Per questa attività, allo scopo di favorire una comprensione graduale dell'imperativo, ho creato un esercizio di cloze A e B in cui si doveva ricavare l'imperativo a partire dall'infinito del verbo (si veda scheda 1 , in appendice). Ogni alunno svolgeva da solo il cloze e poi lo correggeva con il compagno che aveva le soluzioni mancanti. Gli alunni avevano già studiato l'imperativo al primo semestre; si è trattato, quindi, di elicitare le conoscenze pregresse e di approfondire il tema, includendo l'imperativo dei verbi irregolari, la forma negativa e quella con i pronomi. Il cloze facilitato ha permesso di entrare in contatto con la grammatica in maniera ludica e di accedere alla regola generale attraverso la scoperta e il confronto con il compagno. Nell'ultima parte della lezione, a partire dall'esercizio di cloze svolto e coinvolgendo gli alunni, ho proposto una spiegazione globale delle forme e funzioni dell'imperativo. L'attività si è conclusa con il commento del testo letterario (analisi delle strategie linguistiche e letterarie utilizzate) per favorire la comprensione analitica ed esplorativa.

Attività Se una notte d'inverno uno spettatore. Passando dall'immaginario letterario a quello musicale, gli alunni in coppia hanno preparato dei consigli da dare a uno spettatore che sta per ascoltare un concerto, a partire dall' incipit "Stai per cominciare ad ascoltare un concerto...". Potevano riprendere il testo di Calvino come modello e cercare parole nuove su Internet. Infine, hanno letto ad alta voce i testi scritti.

\section{FASE Corpo e movimento (2 ore)}

Presentazione. I task di questa fase sono stati: lavorare sul corpo e sul movimento e utilizzare l'imperativo. Per prima cosa, gli alunni hanno ricostruito il campo lessicale del corpo a partire dalle somiglianze linguistiche con lo spagnolo e il catalano, utilizzando la competenza plurilingue e interculturale. Poi hanno trascritto alla lavagna i nomi delle parti del corpo e hanno lavorato sulle principali differenze con le lingue di provenienza (soprattutto a partire dalla morfologia del plurale irregolare in italiano).

Muovi il tuo corpo. Attraverso la mimica, ho presentato alcuni verbi che indicano movimento e poi ho chiesto loro di riprodurli corporalmente. Per favorire la memorizzazione, si è utilizzato il gioco Muoviti un po', liberamente adattato da Bailini e Consonno (2002, 78-79). In primis, ogni alunno ha dovuto scrivere l'imperativo dei verbi di movimento a partire dall'infinito. L'obiettivo è stato quello di rinforzare il lessico del movimento e del corpo e

19. In effetti, il prologo di Calvino è molto utilizzato nella spiegazione dell'imperativo informale nell'ambito della didattica dell'italiano come LS. Esistono molte attività didattiche che riprendono quest'opera di Calvino. Senza dubbio, è illuminante l'attività didattica Istruzioni per rilassarsi proposta da Accademia del Giglio Blog (s.d.).

20. Per favorire la comprensione del testo ho adattato alcune espressioni al livello A1- A2. 
l'uso dell'imperativo in maniera divertente e ludica per abbassare la tensione e la paura della LS. Ogni alunno ha poi dato delle istruzioni di movimento al compagno, che le ha eseguite fisicamente. Questa parte della sessione si è inspirata al Total Physical Response e ha cercato di combinare parola e azione a partire dall'attività fisica. In un certo senso, il TPR ha permesso di favorire la memorizzazione combinando azione fisica e verbale.

\section{FASE La meditazione dell'albero ( 2 ore)}

Presentazione. Ho portato in classe un cartellone su cui avevo previamente disegnato un albero. Servendosi del dizionario, gli alunni hanno aggiunto sul cartellone i nomi delle varie parti dell'albero.

Meditazione. Questa fase ha rivestito un ruolo centrale all'interno del progetto e si è basata sulla meditazione dell'albero (si veda la scheda 2 , in appendice). ${ }^{21}$ Per svolgere l'attività, gli alunni hanno spostato i banchi e si sono disposti in cerchio a terra a gambe incrociate e con gli occhi chiusi. A partire da una musica di sottofondo, ho letto ad alta voce il testo della meditazione e ho invitato il gruppo a seguire le istruzioni all'imperativo per rilassarsi. La meditazione ha avuto come obiettivi sia l'educazione emozionale (il rilassamento) sia l'educazione linguistica (la comprensione orale dell'imperativo). Dopo l'ascolto, ogni alunno ha letto individualmente il testo e poi ha condiviso le sue sensazioni con il gruppo classe. Questa fase ha presentato vari punti in comune con il metodo suggestopedico: per esempio l'uso della musica e anche l'importanza data agli effetti psicologici del rilassamento per inibire lo stress e creare un ambiente positivo.

Espressione scritta. Il prodotto finale di questa fase è consistito nella scrittura di un breve testo personale da attaccare sul cartellone dell'albero, per favorire la produzione linguistica creativa, poco guidata e poco strutturata. Per facilitare l'espressione scritta, gli alunni hanno riflettuto sulle sensazioni provate a partire dalle domande: 1) con quale parte dell'albero ti identifichi e perché?; 2) come ti senti dopo la meditazione? Durante la stesura del testo, ho chiesto agli alunni di usare il presente indicativo, offrendo un supporto linguistico nella preparazione dei testi. In questo livello linguistico, l'alunno ha spesso notevoli difficoltà nell'espressione scritta perché non ha ancora molto vocabolario. Tuttavia, trattandosi di un esercizio di espressione libera e creativa, i testi scritti hanno mostrato non solo l'acquisizione corretta del vocabolario di questa fase, ma anche l'utilizzo di uno stile poetico, più adeguato all'espressione delle proprie emozioni.

IV FASE Consigli per un giovane musicista (2 ore)

Dare consigli. L'obiettivo di questa fase è stato la produzione di un decalogo di consigli seguendo la struttura dei testi di tipo regolativo, come le istruzioni

21. Si tratta di una meditazione guidata basata sulla visualizzazione: si immagina di visualizzare un oggetto (in genere un elemento della natura) e ci si concentra sulla sua forma, le sue proprietà e il suo significato. 
per una vita sana. Gli alunni in coppia hanno preparato e poi hanno letto ad alta voce dieci consigli generali sul viver bene.

Preparazione del decalogo del musicista. Questa attività è iniziata con un brainstorming alla lavagna a partire dalla domanda: quali sono le istruzioni più importanti per un giovane musicista per suonare bene e godere della sua musica? Agli alunni è stato permesso di consultare gli appunti del project work e di selezionare i consigli più importanti. Inoltre potevano scegliere una musica di sottofondo da accompagnare alla lettura finale del loro testo. In gruppi di quattro, hanno preparato un decalogo con i consigli per un musicista e lo hanno poi presentato al resto della classe.

\section{FASE Autovalutazione (1 ora)}

Scheda di autovalutazione e dibattito finale. Dopo aver compilato una scheda di autovalutazione ${ }^{22}$ individualmente, si è svolto un dibattito finale in gruppo a partire dalle domande:

1) Quali sono state le attività che ti sono piaciute di più??

2) Sei soddisfatto del progetto o no? Spiega il perché...

3) Come si potrebbe migliorare questo progetto?

4) Cosa hai imparato di grammatica, vocabolario, comunicazione?

5) Cosa hai imparato sul tema della mindfulness?

L'intero gruppo classe si è mostrato molto soddisfatto del progetto e ha sottolineato l'importanza di "imparare rilassandosi e senza fretta". In particolare ha apprezzato molto la fase I Se una notte d'inverno uno spettatore e anche la fase III La meditazione dell'albero. Rispetto alle conoscenze acquisite, in molti hanno spiegato di aver appreso non solo l'imperativo, ma anche delle "lezioni di vita". A livello linguistico, gli alunni hanno messo in risalto che il progetto li ha aiutati a sentirsi più sicuri con l'italiano e a superare la paura della lingua straniera. Un'alunna ha voluto spiegare che in questo modo l'imperativo si è rivelato un argomento non solo stimolante, ma anche "fondamentale per viver bene". Inoltre, alcuni alunni hanno aggiunto che durante il progetto hanno imparato un nuovo modo di approcciarsi alla musica attraverso la meditazione.

\section{Conclusioni}

In questo contributo si è visto come, attraverso l'uso di tecniche meditative e creative, gli alunni hanno potuto acquisire la conoscenza delle forme imperativali in maniera efficace, superando le difficoltà presenti in questo livello e focalizzando l'importanza nozionale e comunicativa dell'imperatività in italiano (Berretta, 1995).

Per quanto riguarda le limitazioni di questa ricerca, intese come spunti di riflessione per progetti futuri, metterei in risalto che si sarebbe potuto svolgere un progetto interdisciplinare in collaborazione con altre discipline curricolari;

22. Per formulare il questionario, ho liberamente adattato le domande di autovalutazione sul project work proposte da Mugnani (2011, p. 63). 
inoltre, rispetto al prodotto finale si sarebbe potuta ampliare la tipologia dei lavori presentati favorendo ancora di più la creatività degli alunni (oltre ai testi scritti, la preparazione di materiale audio, video, canzoni, ecc...). Nonostante questo, la proposta di lavorare con una catena di task attraverso il project work è stata comunque molto proficua e si è mostrata adeguata al target della scuola, come ha dimostrato l'autovalutazione finale in cui gli apprendenti hanno espresso un considerevole entusiasmo.

Sia l'approccio comunicativo che quello umanistico-affettivo hanno permesso di spostare il focus dell'attenzione sull'alunno e sui suoi bisogni linguistici ed educativi. In questo senso il progetto ha cercato di intessere una "trama creativa”, per riprendere la felice metafora utilizzata da Ribè (1997). Il project work ha consentito di lavorare sempre con almeno un obiettivo duplice: da una parte le competenze comunicative e dall'altra quelle personali e sociali e tutto ciò che si può acquisire attraverso un uso reale della lingua in gruppo. In questo modo la didattica progettuale ha dimostrato come mettere in crisi il curricolo lineare e ripartire dal lavoro sulle competenze (Quartapelle, 2009).

Lavorare sulla mindfulness ha permesso di implementare allo stesso tempo la consapevolezza, la creatività e anche la componente motivazionale. Gli alunni hanno acquisito perfettamente l'imperativo informale e hanno anche imparato a rilassarsi e a meditare, mettendo in pratica la rule of forgetting teorizzata da Krashen e Terrell (1983). Pertanto la mindfulness può svolgere un ruolo chiave nell'ambito dell'educazione linguistica riuscendo ad abbassare il filtro affettivo, a suscitare emozioni piacevoli e a promuovere l'apprendimento significativo e vivenziale. 


\section{Appendice}

\section{Scheda 1 Esercizio di cloze Se una notte d'inverno un viaggiatore Italo Calvino}

Leggi il testo e trasforma all'imperativo informale tutti $i$ verbi numerati all'infinito. Attenzione: nel testo ci sono altri imperativi informali numerati, prova a dedurre da questi verbi la regola di formazione dell'imperativo. Infine, confrontati con il tuo compagno che ha le soluzioni.

\section{Cloze A . Se una notte d'inverno un viaggiatore Italo Calvino}

Stai per cominciare a leggere il nuovo romanzo Se una notte d'inverno un viaggiatore di Italo Calvino.

1

2 Raccogliti, 3 (rilassarsi).

\section{Respira.}

5 Sentiti libero di godere questo momento. 6 Lascia che il mondo che ti circonda sfumi nell'indistinto. La porta è meglio chiuderla; di là c'è sempre la televisione accesa. 7 (dire) subito, agli altri: "No, non voglio vedere la televisione!" 8 Alza la voce, se no non ti sentono: "Sto leggendo! Non voglio essere disturbato!".

Forse non ti hanno sentito, con tutto quel chiasso; 9 più forte,

10 (gridare): "Sto cominciando a leggere il nuovo romanzo di Italo Calvino!"

$\mathrm{O}$ se non vuoi 11 non dire nulla; speriamo che ti lascino in pace. 12 (prendere) la posizione più comoda: seduto, sdraiato, raggomitolato, coricato. Coricato sulla schiena, su un fianco, sulla pancia. In poltrona, sul divano, sulla sedia a dondolo, sulla sedia a sdraio, sul pouf. Sull'amaca, se hai un'amaca. Sul letto, naturalmente, o dentro il letto. Puoi anche metterti a testa in giù, in posizione yoga, col libro capovolto, si capisce. [...]

Bene, cosa aspetti? 13 Distendi le gambe, 14 (allungare) pure i piedi su un cuscino, su due cuscini, sui braccioli del divano, sul tavolino da tè, sulla scrivania, sul pianoforte, sul mappamondo. 15 Togliti le scarpe, prima. Se vuoi tenere i piedi sollevati; se no, 16 (rimettere) le scarpe. Adesso 17 non restare lì con le scarpe in una mano e il libro nell'altra. 18 (regolare) la luce in modo che non ti stanchi la vista. 19 Fa' queste cose adesso, perché appena sprofondi nella lettura non potrai smuoverti. $\underline{20}$ (fare) in modo che la pagina non resti in ombra, un addensarsi di lettere nere su sfondo grigio; ma 21 sta' attento che non le batta addosso una luce troppo forte [...]. 22 (cercare) di prevedere ora tutto ciò che può interrompere la lettura. [...]. 


\section{Cloze B . Se una notte d'inverno un viaggiatore Italo Calvino}

Stai per cominciare a leggere il nuovo romanzo. Se una notte d'inverno un viaggiatore di Italo Calvino.

1 Rilassati.

2 (raccoglier-si), 3 allontana da te ogni altro pensiero.

$4 \quad$ (respirare).

5 (sentir-si) libero di godere questo momento.

$6 \quad$ (lasciare) che il mondo che ti circonda sfumi nell'indistinto. La porta è meglio chiuderla; di là c'è sempre la televisione accesa. 7 Di' subito, agli altri: "No, non voglio vedere la televisione!". 8 (alzare) la voce, se no non ti sentono: "Sto leggendo! Non voglio essere disturbato!".

Forse non ti hanno sentito, con tutto quel chiasso; 9 parla più forte, 10 grida: "Sto cominciando a leggere il nuovo romanzo di Italo Calvino!".

O se non vuoi 11

(non dire) nulla; speriamo che ti lascino in pace.

12 Prendi la posizione più comoda: seduto, sdraiato, raggomitolato, coricato. Coricato sulla schiena, su un fianco, sulla pancia. In poltrona, sul divano, sulla sedia a dondolo, sulla sedia a sdraio, sul pouf. Sull'amaca, se hai un'amaca. Sul letto, naturalmente, o dentro il letto. Puoi anche metterti a testa in giù, in posizione yoga, col libro capovolto, si capisce. [...]

Bene, cosa aspetti? 13

(distendere) le gambe, 14 allunga pure $\mathrm{i}$ piedi su un cuscino, su due cuscini, sui braccioli del divano, sul tavolino da tè, sulla scrivania, sul pianoforte, sul mappamondo.

15 (toglier-si) le scarpe, prima. Se vuoi tenere i piedi sollevati; se no, 16 rimetti le scarpe. Adesso 17 (non restare) lì con le scarpe in una mano e il libro nellıaltra. 18 Regola la luce in modo che non ti stanchi la vista. 19

(fare) queste cose adesso, perché appena sprofondi nella lettura non potrai smuoverti. $20 \mathrm{Fa}$ in modo che la pagina non resti in ombra, un addensarsi di lettere nere su sfondo grigio; ma 21 (stare)_attento che non le batta addosso una luce troppo forte [...]. 22 Cerca di prevedere ora tutto ciò che può interrompere la lettura. [...].

\section{Scheda 2 La meditazione dell'albero ${ }^{23}$}

Immagina di trovarti in una foresta, un'immensa e rigogliosa foresta.

Che tipo di albero vuoi essere, come sono i tuoi rami, il tuo tronco, le tue radici?

Mettiti in piedi diritto. Respira e rilassati.

Inspira con il naso ed espira con la bocca.

Senti come il respiro si fa sempre più profondo e leggero.

23. Le origini della meditazione dell'albero (come di molte meditazioni) si perdono nella tradizione orale orientale e pertanto ne esistono molte versioni diverse. Ricordo anche che l'albero è un simbolo fondamentale della filosofia orientale e dello yoga e rappresenta l'equilibrio e il radicamento nel presente. Nello specifico, il testo di questa meditazione è liberamente tratto e adattato da Mendez (s.d.). 
Inspira ed espira profondamente.

Chiudi gli occhi.

Concentrati sul tuo respiro.

Inspira con il naso ed espira con la bocca.

Prenditi il tempo di cui hai bisogno.

Inizia a visualizzare il tuo albero.

Ti circonda una foresta rigogliosa e lussureggiante.

Senti l'energia che si espande dal tuo albero.

Adesso siediti a gambe incrociate.

Metti le mani sulle ginocchia, la schiena diritta.

Lascia la bocca semiaperta per respirare.

Continua a inspirare ed espirare con lentezza,

assapora l'aria che entra attraverso i tuoi rami.

Senti il tuo respiro: come entra e invade i polmoni e come esce fuori.

Inspira ed espira lentamente.

Lascia che i pensieri e le preoccupazioni vadano via.

Ripassa la tua giornata e lascia andar via ogni emozione, pensiero, preoccupazione.

Prendi contatto con la parte più profonda di te e, semplicemente, sii presente. Senti come il tuo corpo si rilassa: i capelli, il viso, il collo, il petto, la pancia, le braccia, le mani, le dita, le gambe, le ginocchia, le caviglie, i piedi.

Continua a inspirare ed espirare profondamente.

Ora tutto il tuo corpo è un albero. Sentiti albero.

Sei parte di una foresta immensa che ricopre una montagna.

Senti le tue radici che sprofondano nella terra. Le radici servono a fissarti al terreno, a renderti solido e sicuro, a nutrirti.

Queste radici prelevano l'acqua dal terreno: il nutrimento di cui hai bisogno per crescere e vivere. Un fiume sotterraneo scorre sotto le tue radici. Goditi questa sensazione dell'acqua attraverso le radici.

Le radici sono la tua parte profonda, nascosta. Senti come attraverso le radici l'acqua apporta energia a tutto il tuo corpo dal basso verso l'alto.

Ora senti il tuo tronco. Sei il tronco. Senti com’è: alto, basso, tozzo, sottile, dritto o contorto. Senti la forza del tuo tronco.

Percepisci la corteccia che ti protegge. La corteccia ti permette di respirare e ti protegge dalla pioggia, dai venti.

Senti anche la linfa che scorre al tuo interno fin nel tuo cuore. Senti come la linfa ti nutre.

Percepisci quello che c'è intorno a te.

Senti gli altri alberi, senti se c'è poco o molto spazio intorno a te, se vivi in una foresta piena di altri alberi simili a te o se sei isolato su una collina, se fai parte di un filare ordinato.

Senti anche i tuoi rami, la parte di te che si espande verso l'alto, verso il cielo. Il punto dove nascono foglie, fiori, dove crescono i frutti, dove gli animali fanno la loro casa.

Tu sei l'intero albero in ogni sua parte. 
Percepisci la sensazione di essere un insieme di parti diversissime tra loro: radici, acqua, linfa, semi, corteccia, frutti, foglie, fiori...

Senti come sei profondamente connesso con ciò che ti circonda.

Gli animali si nutrono grazie a te o grazie a te trovano rifugio.

Il ciclo annuale del sole, della luce e delle stagioni è vitale per te.

Resta, per un po', albero, e sentiti parte della natura.

Inspira ed espira profondamente. Come un albero.

(Pausa di qualche minuto)

Ora, lentamente ritorna al tuo corpo: i piedi, le caviglie, le ginocchia, le gambe, il tronco, la pancia, il petto, le braccia, le mani, le dita, il collo, la testa, i capelli.

Lascia che il respiro entri profondamente in te.

Inspira con il naso ed espira con la bocca.

Inizia a muovere le dita delle mani e dei piedi.

Poco a poco riprendi contatto con tutto il tuo corpo.

Ora, quando ti senti pronto, poco a poco puoi riaprire gli occhi con lentezza.

\section{Bibliografia}

Accademia del Giglio Blog (s.d.). Istruzioni per rilassarsi. Disponibile su https://www. adgblog.it/2008/01/18/istruzioni-per-rilassarsi-1-attivita-didattica-per-il-rinforzodellimperativo/

Alan, B., \& Stoller, F. (2005). Maximizing the Benefits of Project Work in Foreign Language Classrooms. English Teaching Forum, 43 (4), 10-21.

Ardissino, A., \& Stroppa, S. (2001). Leggere testi letterari. Torino: Paravia.

Asher, J. (1969). The Total Physical Response Approach to Second Language Learning. The Modern Language Journal, 53 (1), 3-17.

Ausubel, D. P. (1963). The Psychology of Meaningful Verbal Learning. New York: Grune \& Stratton.

Bachman, L. F. (1990). Fundamental Considerations in Language Testing. Oxford: Oxford University Press.

Baer, R.A., \& Maffei, C. (Edd). (2012). Come funziona la mindfulness. Teoria, ricerca, strumenti. Milano: Raffaello Cortina Editore.

Bailini, S., \& Consonno, S. (2002). Ricette per parlare. Attività e giochi per la produzione orale. Firenze: Alma Edizioni.

Balboni, P.E. (2002). Le sfide di Babele. Insegnare le lingue nelle società complesse. Torino: UTET Libreria.

Balboni, P. E (2017). La glottodidattica umanistica in Italia: una prospettiva storica. Educazione linguistica. Language Education El.le, 1, 7-22. doi: http://doi. org/10.14277/2280-6792/ELLE-6-1-17-1

Berretta, M. (1995). Imperativi in italiano: il ruolo della marcatezza pragmatica e morfologica nell'apprendimento di L2. In A. Giacalone Ramat \& G. Crocco Galèas (Edd.), From Pragmatics to Syntax. Modality in Second Language Acquisition (pp. 333-348). Tübingen: Gunter Narr Verlag.

Birello, M., Odelli, E., \& Vilagrasa, A. (2017). A lezione con i task: fra teoria e operatività. Educazione linguistica. Language Education, El.le, 6 (2), 199-215. doi: http:// doi.org/10.30687/2280-6792/2017/02/001 
Brady, R. (2007). Learning to Stop, Stopping to Learn: Discovering the Contemplative Dimension in Education. Journal of Transformative Education, 5 (4), 372-394. doi: https://doi.org/10.1177/1541344607313250

Breen, M. P. (1987). Learner Contributions to Task Design. In C. Candlin \& D. Murphy (Edd.), Language Learning Tasks (pp. 23-46). Englewood Cliffs NJ.: Prentice-Hall.

Bruner, J. (1966) Toward a Theory of Instruction. Cambridge (MA): Harvard University Press.

Calvino, I. (1994) Se una notte d'inverno un viaggiatore. Milano: Mondadori. (1a ed. 1979)

Canale, M. (1983). From Communicative Competence to Communicative Language Pedagogy. In J.C. Richards \& R.W. Schmidt (Edd.), Language and Communication (pp. 2-27). Harlow: Longman.

Canale, M., \& Swain, M. (1980). Theoretical Base of Communicative Approaches to Second Language Teaching and Testing. Applied Linguistics, 1 (1), 1-47.

Caon, F. (2005). Un approccio umanistico affettivo all'insegnamento dell'italiano a non nativi. Venezia: Cafoscarina.

Cardona, M. (2010). L'approccio cognitivo-emozionale e il visconte dimezzato. In F. Caon (Ed.), Facilitare l'apprendimento dell'italiano L2 e delle lingue straniere (pp. 26-38). Torino: UTET Università.

Ceragioli, M. (2005). I soggetti attivi nel progetto. In F. Quartapelle (Ed.), La didattica per progetti (pp. 89-107). Milano: Franco Angeli.

Consiglio d'Europa (2002). Quadro comune europeo di riferimento per le lingue: apprendimento, insegnamento, valutazione (F. Quartapelle \& D. Bertocchi, Tradd.). Firenze: La Nuova Italia.

Departament d'Educació de la Generalitat de Catalunya (2007a). Currículum d'Educació Secundària Obligatòria. Àmbit de llengues (Decret 143/2007 DOGC núm. 4915). Disponibile su http://www.xtec.cat/alfresco/d/d/workspace/SpacesStore/d5234a10-5b77-4215-9938-d481d0ad28d2/llengues_eso.pdf

Departament d'Educació de la Generalitat de Catalunya (2007b). Decret Ordenació dels ensenyaments de l'Educació Secundària Obligatòria. Disponibile su http:// www.xtec.cat/alfresco/d/d/workspace/SpacesStore/5c48aeb8-c20e-4b87-b53ea6e215618672/presentacio_curriculum_eso.pdf

Dewey, J. (1916). Democracy and Education: An Introduction to the Philosophy of Education. New York: MacMillan.

Fallah, N. (2016). Mindfulness, Coping Self-Efficacy and Foreign Language Anxiety: a Mediation Analysis. Educational Psychology, 37 (6), 745-756. doi: https://doi. org/10.1080/01443410.2016.1149549

Freddi, G. (1970). Metodologia e didattica delle lingue straniere. Bergamo: Minerva Italica.

Fried-Booth, D. (1986). Project Work. Oxford: Oxford University Press.

Galli De' Paratesi, N. (1981). Livello soglia per l'insegnamento dell'italiano come lingua straniera. Strasburgo: Consiglio d'Europa.

Ghanizadeh, A., Makiabadi, H. \& Abdi Navokhi, S. (2019). Relating EFL University Students' Mindfulness and Resilience to Self-Fulfilment and Motivation in Learning. Issues in Educational Research, 29 (3), 695-714. Disponibile su http://www. iier.org.au/iier29/ghanizadeh.pdf

Goleman, D. (1995). Emotional Intelligence. London: Bloomsbury. 
Hassed, C., \& Chambers, R. (2015). Mindful Learning: Reduce Stress and Improve Brain Performance for Effective Learning. Boston, MA: Shambhala.

Henry, J. (1994). Teaching Through Projects. London: Kogan Page.

Houston T., \& Turner P. K. (2007). Mindfulness and Communicative Language Teaching. Academic Exchange Quarterly, 11 (1), 138-42. Disponibile su https://www. academia.edu/10087247/Mindfulness_and_communicative_language_teaching

Hymes, D. (1971). On Communicative Competence. Philadelphia: University of Pennsylvania Press.

Institut Escola Artìstic Oriol Martorell (2010). Projecte educatiu de Centre. Disponibile su https://docs.google.com/file/d/0B3Jkdv2MAPF7bTdOb0FjV2p3akk/ edit?pli $=1$

Kabat-Zinn J. (1990). Full Catastrophe Living: Using the Wisdom of Your Body and Mind to Face Stress, Pain, and Illness. New York: Delacorte Press.

Kilpatrick, W. H. (1918). The Project Method. Teachers' College Record, 19 (4), 319-335.

Krashen, S.D. (1981). Second Language Acquisition and Second Language Learning. Oxford: Pergamon Press.

Krashen, S.D., \& Terrel, T. (1983) The Natural Approach: Language Acquisition in the Classroom. Hayward, CA: Alemany Press.

Legutke, M., \& Thomas, H. (1991). Process and Experience in the Language Classroom. London: Longman.

Lozanov, G. (1978). Suggestology and Outlines of Suggestopedy. New York: Gordon and Breach.

Mendez, K. (s.d.). Tree Meditation (Archives for Meditation, Stanford MedicineMindfulness Program). Disponibile su: https://mindful.stanford.edu/2015/04/ tree-meditation/

Mugnani, C. (2011). La didattica progettuale. Le buone pratiche. Perugia: Guerra Edizioni.

Nunan, D. (2004). Task-Based Language Teaching. Cambridge: Cambridge University Press.

Önem, E. E. (2015). A Study on the Effects of Meditation on Anxiety and Foreign Language Vocabulary Learning. Journal of Language and Literature Education, (15), 134-148. Disponibile su https://files.eric.ed.gov/fulltext/ED573228.pdf

Preti, L., Bertocchi, D., \& Quartapelle, F. (2002). Oltre il curricolo lineare. Scuola e Città, (1), 93-110.

Quartapelle, F. (Ed.) (2005). Didattica per progetti. Milano: Franco Angeli. (1a ed. 1999)

Quartapelle, F. (2009). Il progetto: uno strumento pedagogico per curricoli flessibili. Italiano L2 in classe, 1, 8-13. Disponibile su https://www.mondadorieducation.it/risorse/media/sito_italianoperstranieri/pdf/ilsa_rivista_1.pdf.

Ribé, R. (1997). Tramas creativas y aprendizaje de lenguas: prototipos de tareas de tercera generación. Barcelona: Publicacions Universitat de Barcelona.

Ribé, R., \& Vidal, N. (1993). Project Work Step by Step. Oxford: Heinemann.

Richards, J. C. \& Rodgers, T. S. (2001). Approaches and Methods in Language Teaching. Cambridge: Cambridge University Press.

Ridarelli, G. (1998). Project Work. In C. Serra Borneto (Ed.), C'era una volta un metodo. Tendenze attuali della didattica delle lingue straniere (pp. 173-187). Roma: Carocci. 
Roeser R. W., \& Peck S. C. (2009). An Education in Awareness: Self, Motivation, and Self-Regulated Learning in Contemplative Perspective. Educational Psychologist, 44, 119-136. doi: https://doi.org/10.1080/00461520902832376

Rogers, C. (1969). Freedom to Learn. Columbus: Merrill.

Spinelli, B., \& Parizzi, F. (2010). Profilo della lingua italiana. Livelli di riferimento del QCER A1, A2, B1, B2. Firenze: La Nuova Italia.

Stoller, F. (1997). Project Work: A Means to Promote Language Content. English Language Teaching Forum, 35 (4), 2-10.

Titone, R. (1971). Psicolinguistica applicata: introduzione psicologica alla didattica delle lingue. Roma: Armando.

Wang, Y., \& Liu, C. (2016). Cultivate Mindfulness: A Case Study of Mindful Learning in an English as a Foreign Language Classroom. The IAFOR Journal of Education, 4 (2), 141-165. doi: https://doi.org/10.22492/ije.4.2.08 